\title{
Efficacy and Safety of Qinpi Tongfeng Formula Combined with Bloodletting Therapy in the Treatment of Acute Gouty Arthritis: A Study Protocol for a Randomized Controlled Trial
}

\author{
Hang Lu $\mathbb{D},{ }^{1,2}$ Wei Liu $\mathbb{D}^{1,2}$ Yihua Fan $\mathbb{D}^{1,2}$ Wenliang Lv, ${ }^{3}$ Danna Yang, ${ }^{4}$ Chunliu Liu, ${ }^{1,2}$ \\ and Fangfang Lin $^{1,2}$ \\ ${ }^{1}$ First Teaching Hospital of Tianjin University of Traditional Chinese Medicine, Tianjin 300193, China \\ ${ }^{2}$ National Clinical Research Center for Chinese Medicine Acupuncture and Moxibustion, Tianjin 300381, China \\ ${ }^{3}$ Guanganmen Hospital, China Academy of Chinese Medical Sciences, Beijing 100053, China \\ ${ }^{4}$ Gansu University of Chinese Medicine, Lanzhou 730050, Gansu, China
}

Correspondence should be addressed to Wei Liu; fengshiliuwei@163.com

Received 26 August 2021; Accepted 20 December 2021; Published 21 January 2022

Academic Editor: Samuel Silvestre

Copyright $(92022$ Hang Lu et al. This is an open access article distributed under the Creative Commons Attribution License, which permits unrestricted use, distribution, and reproduction in any medium, provided the original work is properly cited.

Background. Acute gouty arthritis (AGA) is a common arthritis disease, with the characteristics of acute onset, severe condition, and poor prognosis. The conventional treatments have shown certain curative effects but are accompanied with many adverse reactions. The combination of orally taken Qinpi Tongfeng Formula (QPTFF) and bloodletting therapy could effectively alleviate arthralgia and joint swelling in AGA patients. However, there is a lack of high-quality randomized controlled trials (RCTs) to evaluate the clinical efficacy and safety of the combined therapy against AGA. Methods. This is a prospective, randomized, parallel controlled trial conducted in the First Teaching Hospital of Tianjin University of Traditional Chinese Medicine to explore the efficacy and safety of QPTFF combined with bloodletting therapy in the treatment of AGA. Eighty-six AGA patients meeting the inclusion and exclusion criteria will be randomly divided into the treatment group and control group in a $1: 1$ ratio using a randomization table. The investigators and the patients will not be blinded, while the outcome assessors and statisticians will be blinded to the allocation. Patients in the treatment group will take QPTFF and bloodletting therapy simultaneously, while patients in the control group will be instructed to orally take colchicine tablets. The primary outcome is the total effective rate, and the secondary outcomes are the pain changes after the first treatment, pain scores, complete pain relief time, joint symptom scores, TCM syndrome score, and laboratory test. SPSS22.0 will be used for statistical analysis. Discussion. This study will evaluate the clinical efficacy and safety of QPTFF combined with bloodletting therapy in the treatment of AGA, and the results of this study will provide reliable clinical evidence for the clinical use of QPTFF combined with bloodletting in the treatment of AGA. The trial is registered with ChiCTR2100048836.

\section{Introduction}

Gout, a crystal-induced arthritis, is caused by monosodium urate deposition because of purine metabolic disorder and/or reduced uric acid excretion [1]. Due to the disorder of purine metabolism or renal excretion, serum uric acid level increases abnormally, and finally, urate crystal deposition and acute persistent inflammatory reaction appear in the joints [2,3]. Gout can be caused by both genetic factors and environmental factors. With the improvement of people's living standards and the change of diet structure and habits, the prevalence of gout increases largely, and the onset age of gout becomes younger and younger $[4,5]$. According to the statistics of the World Health Organization (WHO), about $3.9 \%$ of people in the world are suffering from gout [6], with the ratio of male to female as $3: 1$ to $4: 1$ [7]. Gout patients are often accompanied by obesity, hypertension, nephropathy, hyperlipidemia, and other diseases, which have a serious burden on the health and economy of patients [8]. 
Acute gout arthritis (AGA) is the acute manifestation of gout, characterized by sudden and severe arthralgia with heat, redness, swelling, and restricted movement. At present, nonsteroidal anti-inflammatory drugs (NSAIDs) and colchicine are recommended as the first-line clinical treatments of AGA [9]. These medicines usually have good short-term efficacy, but long-term use may lead to gastrointestinal reactions, rashes, and even liver and kidney function injury $[9,10]$. Hence, a multimodality therapy with fewer side effects to improve arthralgia and swelling of AGA is urgently needed, especially for some patients allergic to NSAIDs and colchicine.

Gout belongs to the paralysis syndrome (bi zheng) in traditional Chinese medicine (TCM), which is caused by dampness-heat accumulation and qi-blood stasis in the channels [11]. In China, the TCM treatment of gout has a long history. It was first discussed separately in Ge Zhi Yu Lun as early as $1347 \mathrm{AD}$. It has been reported that TCM formula combined with external treatment can alleviate the symptoms of gout quickly and delay the development of gout [12]. Qinpi Tongfeng Formula (QPTFF) is a derived compound prescription from Sheng Ji Zong $\mathrm{Lu}$ in $1117 \mathrm{AD}$ according to the modern clinical application. The formula is composed of Cortex Fraxini (Qin Pi), Rhizoma Coptidis (Huang Lian), Radix Saposhnikoviae (Fang Feng), Semen Plantaginis (Che Qian Zi), Rhizoma Smilacis Glabrae (Tu Fu Ling), Rhizome Dioscoreae Hypoglaucae (Bi Xie), Radix Clematidis (Wei Ling Xian), and Herba Siegesbeckiae (Xi Xian Cao). QPTFF has shown the function of clearing dampness-heat, reducing swelling, and relieving pain [13]. A previous clinical study indicated that QPTFF had the same effect on alleviating arthralgia and joint swelling in AGA patients with an analgesic. Moreover, during the clinical trial, serious adverse reactions such as liver and kidney function damage and allergic dermatitis were not found [14]. Bloodletting therapy, one of the traditional external treatment methods, is the withdrawal of blood from a patient to prevent or cure illness and disease. During the process of bloodletting therapy, the operator uses a needle to prick into the skin at specific acupoints and suck a little blood through cupping to dredge the meridians, promote blood circulation, and relieve pain [15]. Clinical studies have found that bloodletting therapy can improve the local microcirculation and promote inflammatory absorption to relieve arthralgia [16]. However, there is still a lack of highquality clinical study to verify the clinical efficacy of the combination of the two therapies in the treatment of AGA. Hence, in this study, we try to conduct a randomized, controlled trial to analyze the efficacy and safety of QPTFF combined with bloodletting therapy in the treatment of AGA.

\section{Materials and Methods}

2.1. Study Design. This trial is designed as an open-label, prospective, randomized, controlled, and parallel-group study. It will be conducted in the First Teaching Hospital of Tianjin University of Traditional Chinese Medicine from August 1, 2021 to October 30, 2021. Figure 1 shows the research flow chart, and the details of patient follow-up are summarized in Figure 2. The research follows the latest Consolidated Standards of Reporting Trials (CONSORT

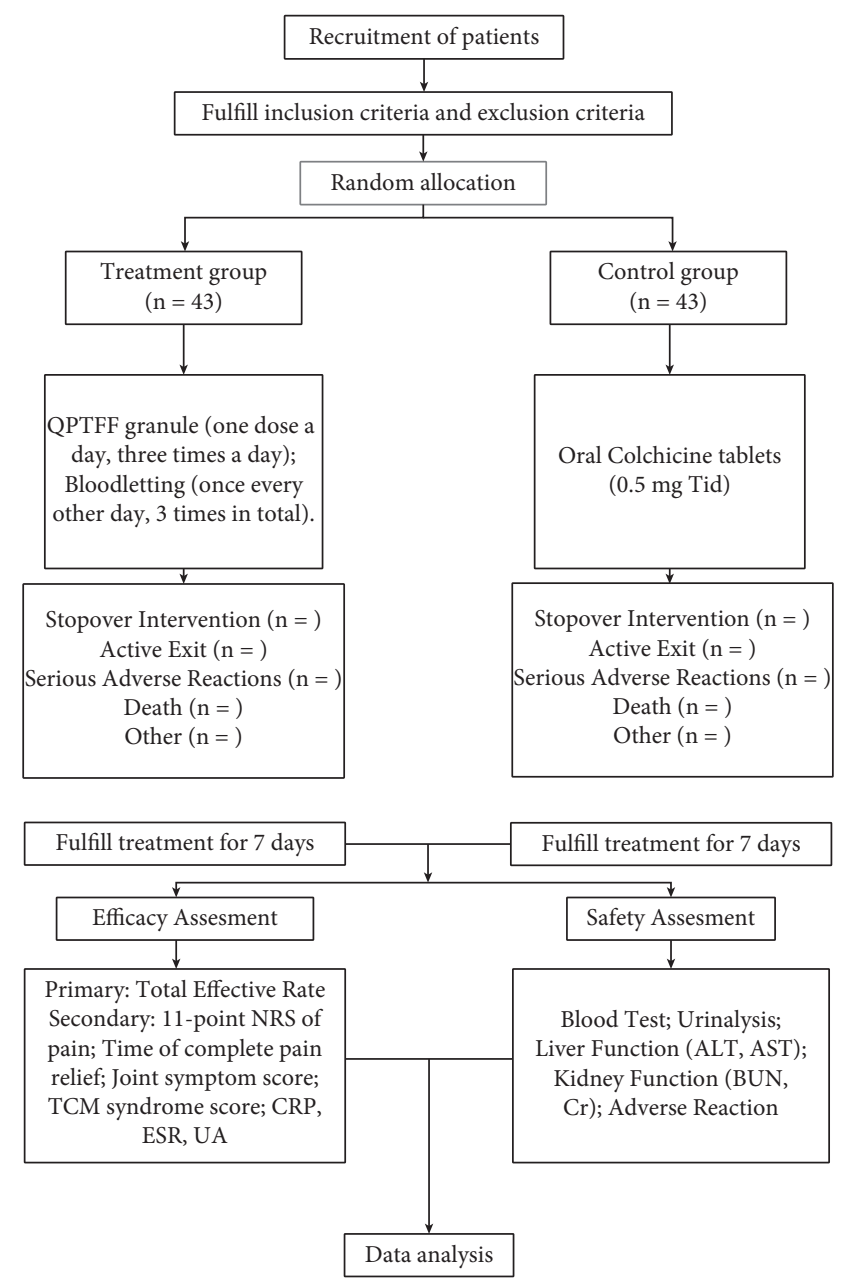

FIgURE 1: The flow chart of this study.

2017) and the Standard Protocol Items: Recommendations for Interventional Trials (SPIRIT) 2013 statement (the SPIRIT checklist is provided in Table S1).

2.2. Ethics and Registration. This study protocol will be conducted in accordance with the Declaration of Helsinki and the Ethical Guidelines for Clinical Research. This study has been approved by the ethics committee of the First Teaching Hospital of Tianjin University of Traditional Chinese Medicine (Ethics number: TYLL2021 [Z] 014), and it has been registered in the Chinese Clinical Trial Registry (Registration number: ChiCTR2100048836).

2.3. Participants. Participants will be recruited from outpatients in the rheumatology department of the First Teaching Hospital in Tianjin University of Traditional Chinese Medicine via posters and WeChat. Recruitment members in the rheumatology department will be in charge of the recruitment and registration of the participants meeting the inclusion and exclusion criteria. Written informed consent will be obtained from participants or their legal representatives, and the personal information will be kept with utmost secrecy. 


\begin{tabular}{|c|c|c|c|c|c|c|c|c|c|}
\hline \multirow[b]{2}{*}{ Project } & $\begin{array}{l}\text { Screening } \\
\text { Period }\end{array}$ & \multicolumn{7}{|c|}{ Treatment Period } & \multirow{2}{*}{$\begin{array}{l}\begin{array}{l}\text { Follow } \\
\text {-up }\end{array} \\
\text { Day } 8\end{array}$} \\
\hline & Baseline & Day 1 & Day 2 & Day 3 & Day 4 & Day 5 & Day 6 & Day 7 & \\
\hline Eligibility screen & $\sqrt{ }$ & & & & & & & & \\
\hline Informed consent & $\sqrt{ }$ & & & & & & & & \\
\hline Randomization & $\sqrt{ }$ & & & & & & & & \\
\hline Treatment & $\sqrt{ }$ & & $\sqrt{ }$ & $\sqrt{ }$ & $\sqrt{ }$ & $\sqrt{ }$ & $\sqrt{ }$ & $\sqrt{ }$ & $\sqrt{ }$ \\
\hline $\begin{array}{l}\text { Combined } \\
\text { medication record }\end{array}$ & & $\sqrt{ }$ & $\sqrt{ }$ & $\sqrt{ }$ & $\sqrt{ }$ & $\sqrt{ }$ & $\sqrt{ }$ & $\sqrt{ }$ & $\sqrt{ }$ \\
\hline $\begin{array}{l}\text { Efficacy } \\
\text { Observation }\end{array}$ & & & & & & & & & \\
\hline $\begin{array}{l}\text { NRS change after } \\
\text { the first treatment }\end{array}$ & $\sqrt{ }$ & $\sqrt{ }$ & & & & & & & \\
\hline NRS & $\sqrt{ }$ & & & & & & & & $\sqrt{ }$ \\
\hline $\begin{array}{l}\text { Complete pain } \\
\text { relief time }\end{array}$ & & & & & & & & & $\sqrt{ }$ \\
\hline $\begin{array}{l}\text { Joint symptom } \\
\text { score }\end{array}$ & $\sqrt{ }$ & & & & & & & & $\sqrt{ }$ \\
\hline $\begin{array}{l}\text { TCM syndrome } \\
\text { score }\end{array}$ & $\sqrt{ }$ & & & & & & & & $\sqrt{ }$ \\
\hline $\begin{array}{l}\text { Laboratory } \\
\text { Indexes }\end{array}$ & $\sqrt{ }$ & & & & & & & & $\sqrt{ }$ \\
\hline $\begin{array}{l}\text { Safety } \\
\text { Evaluation }\end{array}$ & & & & & & & & & \\
\hline $\begin{array}{l}\text { Blood Test and } \\
\text { urinalysis }\end{array}$ & $\sqrt{ }$ & & & & & & & & $\sqrt{ }$ \\
\hline $\begin{array}{l}\text { Liver and kidney } \\
\text { function }\end{array}$ & $\sqrt{ }$ & & & & & & & & $\sqrt{ }$ \\
\hline Adverse event & & $\sqrt{ }$ & $\sqrt{ }$ & $\sqrt{ }$ & $\sqrt{ }$ & $\sqrt{ }$ & $\sqrt{ }$ & $\sqrt{ }$ & $\sqrt{ }$ \\
\hline
\end{tabular}

Figure 2: Patient follow-up process.

2.4. Diagnostic Basis. The diagnosis of AGA is according to the diagnostic criteria of the American College of Rheumatology in 2015 [17]. The syndrome diagnosis of dampness-heat accumulation syndrome in TCM is according to the guidelines for the combined diagnosis and treatment of gout and hyperuricemia issued by the Chinese Society of TCM in 2020 [18], with two primary symptoms or one primary symptom plus two secondary symptoms in combination with the tongue and pulse. The primary symptoms include redness, swelling, heat, and pain in the joint and 
sudden onset of arthralgia, and the secondary symptoms include poor joint movement, fever, and dysphoria. The tongue should be red, with yellow, greasy or thick coating, and the pulse should be slippery.

2.5. Recruitment Criteria. The inclusion criteria are as follows:

(1) Meeting the diagnostic criteria of AGA of the American Society of Rheumatology in 2015

(2) Meeting the TCM diagnostic criteria of gout with dampness-heat accumulation syndrome

(3) 72 hours within the onset of AGA

(4) With moderate, severe, or extreme arthralgia and the score of the numerical rating scale (NRS) $\geq 4$

(5) Without taking any other medicines for AGA 72 hours before the enrollment

(6) Without taking any uric acid-lowering medicines during the last 2 weeks

(7) Age of 18-70 years

(8) With signed informed consent form

2.6. Exclusion Criteria. The exclusion criteria are as follows:

(1) Secondary gouty arthritis caused by other factors (e.g., renal failure, chemotherapy or radiotherapy, and drugs)

(2) With rheumatoid arthritis, psoriatic arthritis, ankylosing spondylitis, knee osteoarthritis, and other arthritis

(3) With multiple joints involved ( $>4$ joints)

(4) Alanine aminotransferase (ALT), alanine aminotransferase (AST), or creatinine (Cr) 1.5 times higher than the upper limit of normal [19]

(5) History of allergy to any ingredients of the medicines in both groups

(6) Women in pregnancy or lactation

(7) With peptic ulcer and bleeding

(8) Having participated in other clinical trials in the past month

(9) With serious mental illness, unable to express accurately or take medicine on time, and unable to cooperate

\subsection{Termination Criteria}

(1) Participants experiencing adverse events (e.g., cardiovascular embolism, gastrointestinal reaction, and severe liver and kidney dysfunction) or other complications that the investigators consider it necessary to terminate trial treatment

(2) Participants could not benefit or even get worse from the trial treatment that the investigators deem necessary to terminate trial treatment
(3) Participants stop or discontinue the medication or take other drugs at will without authorization

(4) Participants are unwilling or impossible to continue the trial and request to withdraw and terminate the trial

2.8. Sample Size. PASS15.0 is used to calculate the sample size. The study is suitable for the noninferiority test. According to our preliminary study, the total effective rate of QPTFF combined with bloodletting is $95 \%$, and the total effective rate of colchicine is $90 \%$ [20]. Taking $\alpha=0.05$, $\beta=0.2$, a ratio of $1: 1$, and the boundary value $=-0.1$, the sample size of the two groups is 78 cases. Considering a loss to follow-up of $10 \%, 86$ cases are finally needed, with 43 cases in each group.

2.9. Randomization and Allocation Concealment. Participants will be randomly divided into the treatment group and control group in a $1: 1$ ratio using a randomization table. The randomization sequence will be generated by an independent statistician with Excel 2013 software. The random numbers will be placed in opaque, sealed envelopes and kept in a safe place until the study is completed. Chunliu Liu will be responsible for the enrollment and assignment of the participants.

2.10. Blinding. Due to the limitation of the intervention program, the blinding of bloodletting therapy is quite difficult to achieve. The investigators and the patients will not be blinded, while the outcome assessors and statisticians will be blinded to the allocation.

2.11. Intervention. In the treatment group, patients will take QPTFF and bloodletting therapy simultaneously. The QPTFF will be provided as granules, composed of Cortex Fraxini (Qin Pi) 30g, Rhizoma Coptidis (Huang Lian) 10g, Semen Plantaginis (Che Qian Zi) 20g, Rhizoma Smilacis Glabrae (Tu Fu Ling) 80g, Radix Clematidis (Wei Ling Xian) 30g, Herba Siegesbeckiae (Xi Xian Cao) 30g, and Radix Saposhnikoviae (Fang Feng) 10g, prepared by Sichuan New Green Pharmaceutical Technology Development Co., Ltd. The patients will be instructed to orally take the granules one dose a day by dissolving them in $50 \mathrm{~mL}$ warm water, three times a day, for 1 week. The bloodletting therapy will be performed by qualified members who have more than 3-year experience in bloodletting therapy after being retrained for standard management for the study. The main acupoints are Ashi points (affected side), SP10 (Xuehai) (bilateral), SP9 (Yinlingquan) (bilateral), ST36 (Zusanli) (bilateral0), BL40 (Weizhong) (bilateral), BL20 (Pishu) (bilateral), and SP6 (Sanyinjiao) (bilateral). At the same time, local points will be selected. For the first metatarsophalangeal joint, SP3 and LR3 will be added; for medial malleolus, KI7 will be added; for the lateral ankle, BL57 will be added. During the bloodletting operation, the patient will take the prone position. The operator disinfects the acupoints with 75\% alcohol, pricks the acupoints with the sterilized lancet, place a 
fire cup on the point for 5 min to suck the blood, and then disinfects the skin with $75 \%$ alcohol. The bloodletting therapy will be performed once every other day, 3 times in total.

In the control group, patients will be instructed to orally take colchicine tablets (Guangdong BIDI Pharmaceutical Co., Ltd., China, H20113208), $0.5 \mathrm{mg}$ once, 3 times a day, for 1 week.

If the pain in the two groups is severe and unbearable during the treatment, etocoxib (Hangzhou MSD Pharmaceutical Co., Ltd., China, J20180059) $120 \mathrm{mg}$, once a day, will be used. The patients will be asked to record the dosage and administration of the medicine.

2.12. Outcome Measures. The primary outcome is the total effective rate according to the Guiding Principles for Clinical Research of New Traditional Chinese Medicine, as divided into clinical cured, remarkably effective, effective, and ineffective [21, 22]. Clinical cured means that the clinical symptoms and signs disappear or basically disappear, and the curative effect index decreases more than 95\% (included). Remarkably effective means that the clinical symptoms and signs are significantly improved, and the curative effect index is reduced more than $60 \%$ (included) but less than 95\%. Effective means improvement of clinical symptoms and signs, and a reduction of curative effect index of more than $30 \%$ (included) but less than $60 \%$. Ineffective means that the clinical symptoms and signs are not improved or even aggravated, and the curative effect index is reduced less than $30 \%$. Total effective rate $=($ clinical cured number + remarkably effective number + effective number)/ total $\times 100 \%$. The total effective rate of the treatment of patients will be evaluated after the treatment by Fangfang Lin.

The secondary outcomes are the pain changes after the first treatment, pain scores, complete pain relief time, joint symptom scores, TCM syndrome score, and laboratory test, as measured by Wenliang Lv and Danna Yang.

(1) The pain changes after the first treatment will be evaluated by the 11-point Numerical Rating Scale (NRS) [23]. The scale includes 0-10 points to represent the different degrees of pain, and the grading standards of pain degree are 0 for painless, $1-3$ for mild pain, 4-6 for moderate pain, and 7-10 for severe pain. In the treatment group, the patients will be instructed to take QPTFF once at the first visit and then to accept bloodletting therapy. After the bloodletting therapy, the changes of NRS scores at $0.5 \mathrm{~h}, 1 \mathrm{~h}, 1.5 \mathrm{~h}$, and $2 \mathrm{~h}$ will be recorded to evaluate the immediate efficacy of TCM therapy. In the control group, after taking colchicine for the first time, the NRS scores of patients will be recorded at $0.5 \mathrm{~h}, 1 \mathrm{~h}, 1.5 \mathrm{~h}$, and $2 \mathrm{~h}$.

(2) Pain scores will be measured by the NRS on day 0 and day 8 (follow-up).

(2) Complete pain relief time is defined as the number of days required from the initial treatment to the complete pain relief measured by the NRS at the end of the study.

(3) Joint symptom score is used to evaluate the tenderness, redness, swelling, and mobility of joints. The higher the score, the heavier the symptom is. The scores will be recorded on day 0 and day 8 (followup).

(4) TCM syndrome score, according to the Guiding Principles for Clinical Research of New Traditional Chinese Medicine [22], includes TCM syndromes such as joint swelling, skin temperature, thirst, and yellow urine. The higher the score, the more serious the condition is. The scores will be recorded on day 0 and day 8 (follow-up).

(5) Laboratory tests include C-reactive protein (CRP), erythrocyte sedimentation rate (ESR), and uric acid (UA). The results will be recorded on day 0 and day 8 (follow-up).

2.13. Safety Evaluation. Blood test, urinalysis, liver function, and renal function (urea nitrogen and serum creatinine) will be measured on day 0 and day 8 to evaluate the safety of treatment. At the same time, patients are required to record any adverse reactions during the study and report to the investigator at any time. Details of all adverse events will be recorded in case report dorms (CRFs), including time, degree and duration, suspected causes, measures, and results. After the treatment, the adverse reactions of the two groups will be counted.

2.14. Data Management and Quality Control. Any modification or change of the protocol will be approved through the formal procedures of the ethics committee of the First Affiliated Hospital of Tianjin University of Traditional Chinese Medicine. Independent clinical research assistants will regularly review the study progress. CRFs will be used in data collection to record demographics, assessment, and reasons for drop-out. All CRFs will be stored in an independent storage room to protect confidentiality. Without the written permission of the supervisor, the participant's information will not be disclosed and shared. At the end of the study, the investigator will submit the CRFs to the data management committee, and the investigators cannot modify the data.

2.15. Statistical Analysis. Efficacy evaluation will be determined by full analysis set (FAS) and per-protocol set (PPS), and safety evaluation will be based on safety set (SS). The statistical evaluation of FAS will follow the intent-to-treat (ITT) principle. The last observation carried forward (LOCF) method will be used to estimate the missing values of main variables. The collected data will be statistically analyzed by using the SPSS22.0 software (International Business Machines Corporation, New York, USA). For continuous data, they will be represented as mean \pm standard deviation and frequency or percentage for categorical data. For the primary outcome, the 
chi-square test will be used. For secondary outcomes, independent samples $T$ test or Mann-Whitney $U$ test for intergroup comparison will be used for pain scores, complete pain relief time, joint symptom scores, TCM syndrome score, and laboratory test and generalized linear models or repeated measures analysis of variance for the pain changes after the first treatment after considering the normality and homogeneity. Statistical testing is two-sided, and $P<0.05$ is considered statistically significant.

\section{Discussion}

The main TCM syndrome of AGA is dampness-heat accumulation syndrome [24], which is characterized by arthralgia, swelling, redness, and fever, accompanied by dysphoria, thirst, and yellow urine [21]. Based on the theory of TCM, the treatment of AGA with dampness-heat accumulation syndrome should follow the principle of clearing dampness-heat and dredging meridians. The mechanism is to inhibit inflammatory factors in the joint fluid, reduce serum uric acid, promote uric acid excretion, regulate immune function, and block peripheral nerve pain [12].

Traditional Chinese medical formula has the characteristics of multicomponent, multitarget, and multilevel in the treatment of AGA [24]. Modern pharmacological studies have found that aescin, the effective component of the main herb Cortex Fraxini (Qin Pi) in QPTFF, can inhibit the overexpression of inflammatory factors and catabolic genes, promote the upregulation of cartilage-specific genes and scavenge reactive oxygen species (ROS), to show anti-inflammatory, antioxidant, and protective effects on chondrocytes [26, 27]. Rhizoma Smilacis Glabrae (Tu Fu Ling) can inhibit uric acid production and promote uric acid excretion, reduce capillary permeability, improve microcirculation, and improve joint swelling [28]. Its effective component, resveratrol, can inhibit IL- $1 \beta$ secretion and downregulation of $\mathrm{NF}-\kappa \mathrm{B}$ p 65 expression and reduce the production of inflammatory factors and chemokines and inflammatory cell infiltration [29]. Herba Siegesbeckiae (Xi Xian Cao) has anti-inflammatory and analgesic effects. It can regulate joint inflammation and pain by controlling inflammatory factors in joints and reducing immune response [30]. Rhizome Dioscoreae Hypoglaucae (Bi Xie) can regulate the expression of inflammatory factors, reduce uric acid in the blood, and protect renal function [31]. In a word, a variety of effective components in QPTFF could play therapeutic roles against AGA.

In China, bloodletting therapy is often used to treat AGA with dampness-heat accumulation syndrome. Clinical studies have found that bloodletting therapy can upregulate the local anti-inflammatory factors IL-4 and IL-10, mediate TLR4/IL-1 signal pathway, regulate immune response, reduce inflammatory cell infiltration [15], and relieve pain. Stimulating BL40 can promote uric acid excretion and improve microcirculation [32], and stimulating SP6 and SP9 can inhibit the synthesis of inflammatory cytokines and reduce TNF- $\alpha$ in serum to play anti-inflammatory and detumescence roles [33]. ST36 could effectively function in reducing inflammation in many studies $[34,35]$, and SP6 can reduce the production and secretion of pain-causing factors and monoamine transmitters in AGA rats to relieve pain $[33,36]$.

The combination of QPTFF and bloodletting therapy has positive and safe effects on AGA patients, but there is still a lack of high-quality clinical study. The previous reported RCTs of TCM in the treatment of AGA showed many limitations. For example, the treatment in the control group was not a classic control such as NASID or colchicine [37]. In addition, those RCTs did not observe the immediate efficacy of TCM treatment [38]. In our study, we try to observe the pain changes within 2 hours right after the patients take the combined therapy, which is helpful to analyze the immediate effect of TCM therapy. Therefore, this study intends to explore the efficacy of QPTFF combined with bloodletting therapy through a high-quality RCT. We take the changes of patients' clinical symptoms and signs as the main outcome indicators, including joint symptoms and TCM syndromes. In addition, we use laboratory indexes to evaluate the efficacy of TCM therapy objectively and liver and kidney function, blood test, and urinalysis to evaluate the safety of the treatment.

Still, there are also some limitations in this study. Due to the characteristics of bloodletting therapy, the operator and the patients cannot be blinded, which may impact the research results. Due to the lifestyle and diet habits of the population in Tianjin, the similar baseline information of the body mass index (BMI), history of gout, and uric acid level in both groups may lead to a single sample and regionalization. Because we just observed the short-term curative effect on AGA, it was difficult to observe the long-term efficacy in two groups after the trial.

\section{Abbreviations}

NSAIDs: Nonsteroidal anti-inflammatory drugs

CONSORT: Consolidated Standards of Reporting Trials

RCT: $\quad$ Randomized controlled trial

QPTFF: Qinpi Tongfeng Formula

ROS: $\quad$ Reactive oxygen species

ALT: $\quad$ Alanine aminotransferase

AST: Alanine aminotransferase

Cr: Creatinine

CRP: $\quad$ C-reactive protein

ESR: $\quad$ Erythrocyte sedimentation rate

UA: Uric acid

NRS: $\quad$ Numerical rating scale

CRF: $\quad$ Case report form

FAS: $\quad$ Full analysis set

PPS: $\quad$ Per-protocol set

SS: $\quad$ Safety set

ITT: Intent-to-treat

LOCF: Last observation carried forward.

\section{Data Availability}

After the study, the datasets used and analyzed in the current study are available from the corresponding author on reasonable request (Wei Liu: fengshiliuwei@163.com). Also, the 
data will be available in the "Chinese Clinical Trial Registry" (http://www.chictr.org.cn). The registration number is ChiCTR2100048836.

\section{Ethical Approval}

This study has been approved by the ethics committee of the First Teaching Hospital of Tianjin University of Traditional Chinese Medicine (TYLL2021 [Z] 014).

\section{Consent}

All the patients participating in the study will sign an informed consent form. Patient privacy and research data will be kept confidential in accordance with clinical trial requirements. The data used for statistical analysis and paper writing will also be anonymously performed with the consent of the patient. The results of this study will be published in the form of a paper.

\section{Disclosure}

Hang Lu, Wei Liu, and Yihua Fan are co-first authors. The funding body had no role in the study design or the decision to submit the manuscript for publication.

\section{Conflicts of Interest}

The authors declare that they have no conflicts of interest.

\section{Authors' Contributions}

Hang Lu and Yihua Fan conceived the study and developed the first trial protocol. Hang Lu, Wei Liu, and Yihua Fan designed the study and drafted the manuscript. Wenliang Lv and Danna Yang guided the research program. Chunliu Liu Fangfang Lin, and Qingxiang $\mathrm{Gu}$ revised the manuscript. Hang Lu, Wei Liu, and Yihua Fan contributed equally to this work.

\section{Acknowledgments}

This study was supported by the National Natural Science Foundation of China (No. 82074377) and the Traditional Chinese Medicine Inheritance and Innovation "Hundred Million" Talent Project (Qihuang Project) (Chinese Medicine People's Education Letter [2018] No. 12)-Liu Wei Qihuang Scholar Studio Construction Project and Tianjin Key Specialty Program (20210602-1).

\section{Supplementary Materials}

Table S1: SPIRIT 2013 checklist: recommended items to address in a clinical trial protocol and related documents. (Supplementary Materials)

\section{References}

[1] N. Dalbeth, T. R. Merriman, and L. K. Stamp, "Gout," The Lancet, vol. 388, no. 10055, pp. 2039-2052, 2016.
[2] K.-H. Yu, D.-Y. Chen, J.-H. Chen et al., "Management of gout and hyperuricemia: multidisciplinary consensus in Taiwan," International Journal of Rheumatic Diseases, vol. 21, no. 4, pp. 772-787, 2018.

[3] F. Sivera, M. D. Wechalekar, M. Andrés, R. Buchbinder, and L. Carmona, "Interleukin-1 inhibitors for acute gout," Cochrane Database of Systematic Reviews, vol. 9, p. Cd009993, 2014.

[4] P. Richette, M. Doherty, E. Pascual et al., "2018 updated European League against Rheumatism evidence-based recommendations for the diagnosis of gout," Annals of the Rheumatic Diseases, vol. 79, no. 1, pp. 31-38, 2020.

[5] L. K. Stamp and N. Dalbeth, "Prevention and treatment of gout," Nature Reviews Rheumatology, vol. 15, no. 2, pp. 68-70, 2019.

[6] H. K. Choi and G. Curhan, "Gout: epidemiology and lifestyle choices," Current Opinion in Rheumatology, vol. 17, no. 3, pp. 341-345, 2005.

[7] J. A. Singh and A. Gaffo, "Gout epidemiology and comorbidities," Seminars in Arthritis and Rheumatism, vol. 50, no. 3s, pp. S11-s16, 2020.

[8] M. M. Elfishawi, N. Zleik, Z. Kvrgic et al., "The rising incidence of gout and the increasing burden of comorbidities: a population-based study over 20 years," Journal of Rheumatology, vol. 45, no. 4, pp. 574-579, 2018.

[9] L. Wilson and J. J. Saseen, "Gouty arthritis: a review of acute management and prevention," Pharmacotherapy: The Journal of Human Pharmacology and Drug Therapy, vol. 36, no. 8, pp. 906-922, 2016.

[10] F. Perez-Ruiz, N. Dalbeth, and T. Bardin, "A review of uric acid, crystal deposition disease, and gout," Advances in Therapy, vol. 32, no. 1, pp. 31-41, 2015.

[11] W.-H. Li, J.-R. Han, P.-P. Ren, Y. Xie, and D.-Y. Jiang, "Exploration of the mechanism of Zisheng Shenqi decoction against gout arthritis using network pharmacology," Computational Biology and Chemistry, vol. 90, p. 107358, 2021.

[12] X. Chi, H. Zhang, S. Zhang, and K. Ma, "Chinese herbal medicine for gout: a review of the clinical evidence and pharmacological mechanisms," Chinese Medicine, vol. 15, no. 1, p. 17, 2020.

[13] W. Liu, Y. H. Wu, L. Zhang et al., "Clinical randomized controlled trial of clearing heat and detoxification and removing dampness and turbidity in the treatment of Gout," China Journal of Traditional Chinese Medicine and Pharmacy, vol. 31, no. 3, pp. 1113-1116, 2016.

[14] W. Liu, Y. H. Wu, B. Xue et al., "Effect of integrated traditional Chinese and Western medicine on Gout," Journal of Traditional Chinese Medicine, vol. 41, no. 5, pp. 1-21, 2021.

[15] R. Li, P. Zhang, Z. Hu et al., "Efficacy and safety of prickingblood therapy for acute gouty arthritis," Medicine, vol. 99, no. 50, p. e23521, 2020.

[16] Y. Zhu, H. W. Yu, Y. Z. Pan, B. Wu, X. Hu, and Y. Cao, "Clinical observation of blood pricking and moxibustion in the treatment of acute gouty arthritis," Chinese Acupuncture \& Moxibustion, vol. 35, no. 9, pp. 885-888, 2015.

[17] T. Neogi, T. L. T. A. Jansen, N. Dalbeth et al., "2015 gout classification criteria: an American college of Rheumatology/ European league against rheumatism collaborative initiative," Annals of the Rheumatic Diseases, vol. 74, no. 10, pp. 17891798, 2015.

[18] Q. Jiang, M. Han, X. P. Tang, C. G. Luo, and X. Gong, "Guidelines for combined diagnosis and treatment of Gout and Hyperuricemia," Journal of Traditional Chinese Medicine, vol. 62, no. 14, pp. 1276-1288, 2021. 
[19] X. N. Yu, H. Y. Wu, Y. P. Deng et al., "“'Yellow-dragon wonderful-seed formula" for hyperuricemia in gout patients with dampness-heat pouring downward pattern: a pilot randomized controlled trial," Trials, vol. 19, no. 1, p. 551, 2018.

[20] S. K. Lu, "Comparison of clinical effects of different doses of colchicine in the treatment of gouty arthritis," Chinese Journal of General Practice, vol. 15, no. 10, pp. 1700-1703, 2017.

[21] Wang, Y. Zhang, M. Dai et al., "Efficacy of Rebixiao Chinese herbal tablets and Chinese formula granules in acute gout arthritis patients: a randomized, multicenter, double-blind, controlled trial," Journal of traditional Chinese Medicine=Chung $i$ tsa chih ying wen pan, vol. 40, no. 4, pp. 664-673, 2020.

[22] D. Xy, Guiding Principles of Clinical Research on New Drugs of Traditional Chinese Medicine, China Pharmaceutical Science and T echnology Press, Beijing, China, 2002.

[23] J. T. Farrar, J. P. Young, L. LaMoreaux, J. L. Werth, and M. R. Poole, "Clinical importance of changes in chronic pain intensity measured on an 11-point numerical pain rating scale," Pain, vol. 94, no. 2, pp. 149-158, 2001.

[24] Z. J. Lin, Z. K. Huang, Y. Wang, T. Yang, and B. Zhang, "Construction and application of research platform on the utility mechanism of traditional Chinese medicine for Gout," World Chinese Medicine, vol. 16, no. 1, pp. 41-45, 2021.

[25] J. M. Li, X. Zhang, X. Wang, Y. C. Xie, and L. D. Kong, "Protective effects of cortex fraxini coumarines against oxonate-induced hyperuricemia and renal dysfunction in mice," European Journal of Pharmacology, vol. 666, no. 1-3, pp. 196-204, 2011.

[26] Y. T. Zhan, Z. N. Qin, and L. Zhen, "Effects of fraxinoside on lipopolysaccharide induced oxidative stress and inflammatory factors in chondrocytes," Journal of Guangxi Medical University, vol. 38, no. 1, pp. 28-34, 2021.

[27] X. L. Sun, K. Y. Wang, and D. Q. Zhang, "Experimental study on anti-inflammatory and analgesic effects of tufuling injection," Chinese Journal of Traditional Medical Science and Technology, vol. 11, no. 4, pp. 231-232, 2004.

[28] T. Wang, X. Y. Zhang, W. Zhang, X. Y. Yang, and Z. F. Li, "Research progress on the mechanism of tufuling in preventing and treating gout," Liaoning Journal of Traditional Chinese Medicine, vol. 48, no. 1, pp. 215-217, 2021.

[29] W. Li, Y. Q. Zhang, H. Li, and W. X, K. D. Zhu, "Research progress on chemical constituents, pharmacological activities and clinical application of Xiqiancao," Journal of Jiangxi University of Traditional Chinese Medicine, vol. 31, no. 4, pp. 102-107, 2019.

[30] Y. Xiao and G. Z. Li, "Research progress on pharmacological effects of bixie," Shanxi Journal of Traditional Chinese Medicine, vol. 34, no. 7, pp. 54-56, 2018.

[31] Y. L. Ma, R. L. Chen, J. Peng, and Z. L. Liu, "Data mining study on clinical acupoint selection law of bloodletting therapy in the treatment of gouty arthritis," Acta Chinese Medicine and Pharmacology, vol. 49, no. 8, pp. 37-41, 2021.

[32] Y. Y. Lin, S. Y. Su, M. X. Qin, and X. Y. Jiang, "Research progress on the mechanism of Acupuncture and moxibustion in the treatment of acute gouty arthritis," Liaoning Journal of Traditional Chinese Medicine, vol. 48, no. 3, pp. 199-202, 2021.

[33] F. M. Yang, Y. N. Gong, N. N. Yu et al., "ST36 acupuncture alleviates the inflammation of adjuvant-induced arthritic rats by targeting monocyte/macrophage modulation," Evidence Based Complementary Alternative Medicine, vol. 2021, Article ID 9430501, 2021.
[34] S. Liu, Z. Wang, Y. Su et al., "A neuroanatomical basis for electroacupuncture to drive the vagal-adrenal axis," Nature, vol. 598, no. 7882, pp. 641-645, 2021.

[35] W. Chai, Y. Tai, X. Shao et al., "Electroacupuncture alleviates pain responses and inflammation in a rat model of acute Gout arthritis," Evidence-Based Complementary and Alternative Medicine: eCAM, vol. 2018, Article ID 2598975, 2018.

[36] Q. Zhang, R. Li, J. Liu et al., "Efficacy and tolerability of Guizhi-Shaoyao-Zhimu decoction in gout patients: a systematic review and Meta-analysis," Pharmaceutical Biology, vol. 58, no. 1, pp. 1023-1034, 2020.

[37] X. Huang, Z. Zhu, G. Wu et al., "Efficacy and safety of external application of Traditional Chinese Medicine for the treatment of acute gouty arthritis: a systematic review and Meta-analysis," Journal of Traditional Chinese Medicine $=$ Chung $i$ tsa chih ying wen pan, vol. 39, no. 3, pp. 297-306, 2019.

[38] S.-J. Zhang, J.-P. Liu, and K.-Q. He, “Treatment of acute gouty arthritis by blood-letting cupping plus herbal medicine," Journal of Traditional Chinese Medicine, vol. 30, no. 1, pp. 18-20, 2010. 